'Departamento de Patología, Instituto Nacional de

Cancerología, México, D.F. 2Departamento de Hematología, Instituto Nacional de

Cancerología, México, D.F.

${ }^{3}$ Facultad de Medicina, Universidad Nacional Autónoma de México.

Los autores no declaran conflicto de interés.

El trabajo no recibió financiamiento.

Recibido el 6 de junio de 2017 , aceptado el 28 de septiembre de 2017.

Correspondencia a: Dr. Alejandro Avilés Salas Departamento de Patología Instituto Nacional de Cancerología.

Av. San Fernando 22, Sección XVI Tlalpan, CP 14080, México, D.F. Tel: (52) 5556280400

Fax: (52) 5556280421 alejandroaviles2001@yahoo.com

\section{Neoplasia blástica de células dendríticas plasmocitoides. Casos clínicos}

\author{
ALEJANDRO AVILÉS-SALAS ${ }^{1,3}$, MYRNA CANDELARIA $^{2}$, \\ MAYRA PONCE ${ }^{2}$, ABRAHAM CAÑAVERA-CONSTANTINO ${ }^{1}$, \\ SEBASTIÁN BURGOS ${ }^{2}$
}

\section{Blastic plasmacytoid dendritic cell neoplasm. Report of three cases}

Blastic plasmacytoid dendritic cell neoplasm is a rare hematological malignancy derived from immature plasmacytoid dendritic cells. The tumor cells have an immature blastic appearance, and diagnosis is based on the expression of CD4, CD56 y CD123 in the absence of other lymphoid, natural killer, or myeloid antigens. The majority of affected individuals are older people with a mean age of 66 years. Male to female ratio is approximately 3:1. Common presentation includes cutaneous lesions followed by tumor dissemination. Treatment with conventional chemotherapy is ineffective and allogeneic hematopoietic stem cell transplantation is required to achieve remission. We report three male patients, aged 23, 27 and 51 years with the disease. All had multiple, infiltrated pink plaques and nodules on the skin of their face, neck and thorax, measuring 1 to $12 \mathrm{~cm}$ in diameter. All tumors were histologically characterized by a monotonous proliferation of medium size cells with blastic features. Tumor cells were positive for CD123, CD56, CD4 and CD7 in all cases. After a mean of follow-up of 14.6 months, one patient died of the disease, one patient is alive and the disease relapsed after 17 months of remission and one patient is alive with no evidence of the disease.

(Rev Med Chile 2017; 145: 1208-1212)

Key words: Dentritic Cells; Hematologic Neoplasms; Killer Cells, Natural.

\section{L} a neoplasia blástica de células dendríticas plasmocitoides (NBCDP) es una entidad relativamente rara que afecta principalmente pacientes adultos del sexo masculino ${ }^{1,2} . \mathrm{Su}$ contraparte normal es la célula dendrítica plasmocitoide de origen hematopoyético, la cual expresa el receptor $\alpha$ de la IL-3 (CD123) en su superficie y carece de marcadores de estirpe mieloide y linfoide. Su función biológica consiste principalmente en establecer un mecanismo de regulación entre la respuesta inmunológica innata y específica ${ }^{3,4}$.

La mayoría de los casos se manifiestan como una enfermedad cutánea en forma de nódulos y/o placas $^{5}$, con compromiso de la médula ósea y/o sangre periférica ${ }^{6}$.

El diagnóstico se establece mediante el estudio histopatológico de las lesiones cutáneas, caracterizado por la proliferación de células neoplásicas de aspecto blástico que infiltran la dermis y en algunos casos el tejido subcutáneo, con expresión de CD4, CD56, CD123, TCL-1 y CD303". El comportamiento biológico es agresivo y muchos pacientes fallecen en un período corto después del diagnóstico ${ }^{3}$.

El objetivo de este estudio es informar las características clínico-patológicas de la NBCDP diagnosticados en el Instituto Nacional de Cancerología de México. 


\section{Presentación de casos}

\section{Caso 1}

Paciente varón de 51 años de edad sin antecedentes relevantes. Inició su enfermedad en mayo de 2014 con diaforesis y fiebre no cuantificada; así como nódulos cutáneos en la cabeza y el tórax. En la exploración física se observaron nódulos violáceos de bordes mal definidos, localizados en la piel de la región frontal y parietal izquierdos, y en el tórax; el mayor de estos midió $2,3 \mathrm{~cm}$. Además se palparon adenopatías bilaterales en el cuello y las axilas. Se realizó biometría hemática que reportó: leucocitos de 18,3 miles $/ \mathrm{mm}^{3}$, linfocitos absolutos de 2,8 miles $/ \mathrm{mm}^{3}$, hemoglobina de $5,2 \mathrm{~g} / \mathrm{dl} \mathrm{y}$ plaquetas de $5 \mathrm{miles} / \mathrm{mm}^{3}$. Biopsia de uno de estos nódulos mostró infiltración predominantemente perianexial no epidermotrópica por neoplasia maligna de células de tamaño intermedio de aspecto blástico (Figura 1A). Se realizó estudio de inmunohistoquímica (IHQ) que resultó positivo en las células neoplásicas para CD123, CD56, CD7 y CD4 (Figuras 1B-1D) y negativo para CD3, CD8, CD20, c-Kit y mieloperoxidasa (MPO). El aspirado y biopsia de médula ósea se informaron infiltradas. El estudio citogenético convencional informó monosomía del cromosoma 13 (-13q14.3) en 75\% de las células analizadas. Se estadificó en etapa IV (Ann Arbor) e inició tratamiento con HiperCVAD (ciclofosfamida, vincristina, doxorrubicina y dexametasona, alternando con dosis altas de metotrexate y citarabina). Posteriormente, recibió trasplante alogénico de células progenitoras hematopoyéticas (TAloCPH) con buena evolución; sin embargo en el día +178 posTAloCPH se documentó recaída en piel de ambos muslos y médula ósea. Finalmente, el paciente falleció con enfermedad a 14 meses del diagnóstico inicial.

\section{Caso 2}

Paciente varón de 27 años de edad que inició su enfermedad en junio de 2015 con aparición de nódulo cutáneo hiperpigmentado de bordes mal definidos en la región del abdomen, el cual creció de manera progresiva hasta alcanzar $12 \mathrm{~cm}$ de eje mayor. Posteriormente, aparecieron múltiples lesiones cutáneas localizadas en la cara, el tórax y las extremidades superiores. A su ingreso se observaron lesiones cutáneas violáceas de bordes irregulares que ocupaban $70 \%$ de la superficie corporal. La biometría hemática reportó: leucocitos de $22,5 \mathrm{miles} / \mathrm{mm}^{3}$, linfocitos absolutos de 16,5 miles $/ \mathrm{mm}^{3}$, hemoglobina de $14,4 \mathrm{~g} / \mathrm{dl}$ y plaquetas de $68 \mathrm{miles} / \mathrm{mm}^{3}$. El aspecto de las lesiones variaba desde máculas y placas hasta nódulos exofíticos; la mayor se localizó en la fosa iliaca derecha y midió $12 \mathrm{~cm}$. Se tomó biopsia de una de ellas y el estudio histopatológico mostró infiltración difusa de la dermis por neoplasia maligna de células de tamaño pequeño a intermedio de aspecto blástico (Figura 1E ). El estudio de IHQ resultó positivo en las células neoplásicas para CD123, CD56, CD4 y CD7 (Figuras $1 \mathrm{~F}-1 \mathrm{H}$ ) y negativo para CD3, CD8, CD20, CD30, CD34, c-Kit y MPO. El aspirado y biopsia de médula ósea se informaron infiltradas. Se estadificó en etapa IV (Ann Arbor) e inició tratamiento con HiperCVAD. Durante su evolución presentó fiebre y se documentó Staphylococcus aureus que se manejó de manera satisfactoria con dicloxacilina. Posteriormente recibió TAloCPH con buena evolución y recuperación hematológica; sin embargo, a 17 meses de seguimiento se documentó recaída en piel y médula ósea.

\section{Caso 3}

Paciente varón de 23 años de edad que inició su enfermedad 11 meses previos a su ingreso con lesión cutánea en la región frontal de bordes mal definidos, exofítica y ulcerada. Se tomó biopsia incisional de la lesión y fue referido con el diagnóstico de rabdomiosarcoma. A su ingreso se observó dermatosis diseminada constituida por placas eritematosas que involucraba la frente, los pómulos, el tórax, los hombros y las caras dorsales de ambos brazos; la mayor se localizó en la frente y midió $8 \mathrm{~cm}$. Se realizó biometría hemática que reportó: leucocitos de $22,5 \mathrm{miles} / \mathrm{mm}^{3}$, linfocitos absolutos de $16,5 \mathrm{miles} / \mathrm{mm}^{3}$, hemoglobina de $14,4 \mathrm{~g} / \mathrm{dl}$ y plaquetas de $68 \mathrm{miles} / \mathrm{mm}^{3}$. En el estudio histopatológico del material de revisión, se observó infiltración difusa de la dermis y el tejido subcutáneo por neoplasia maligna de células de tamaño intermedio de aspecto blástico, con elevado índice mitósico (Figura 1I ). Se realizó estudio de IHQ que resultó positivo en las células neoplásicas para CD123, CD56, CD4 y CD7 (Figuras 1J-1L ) y negativo para CD3, CD8, CD20, CD30 y MPO. El aspirado y biopsia de médula ósea se reportaron sin infiltración. Recibió tratamiento con HiperCVAD y posteriormente TAloCPH. Actualmente el paciente se encuentra vivo sin actividad tumoral a 13 meses de seguimiento. 


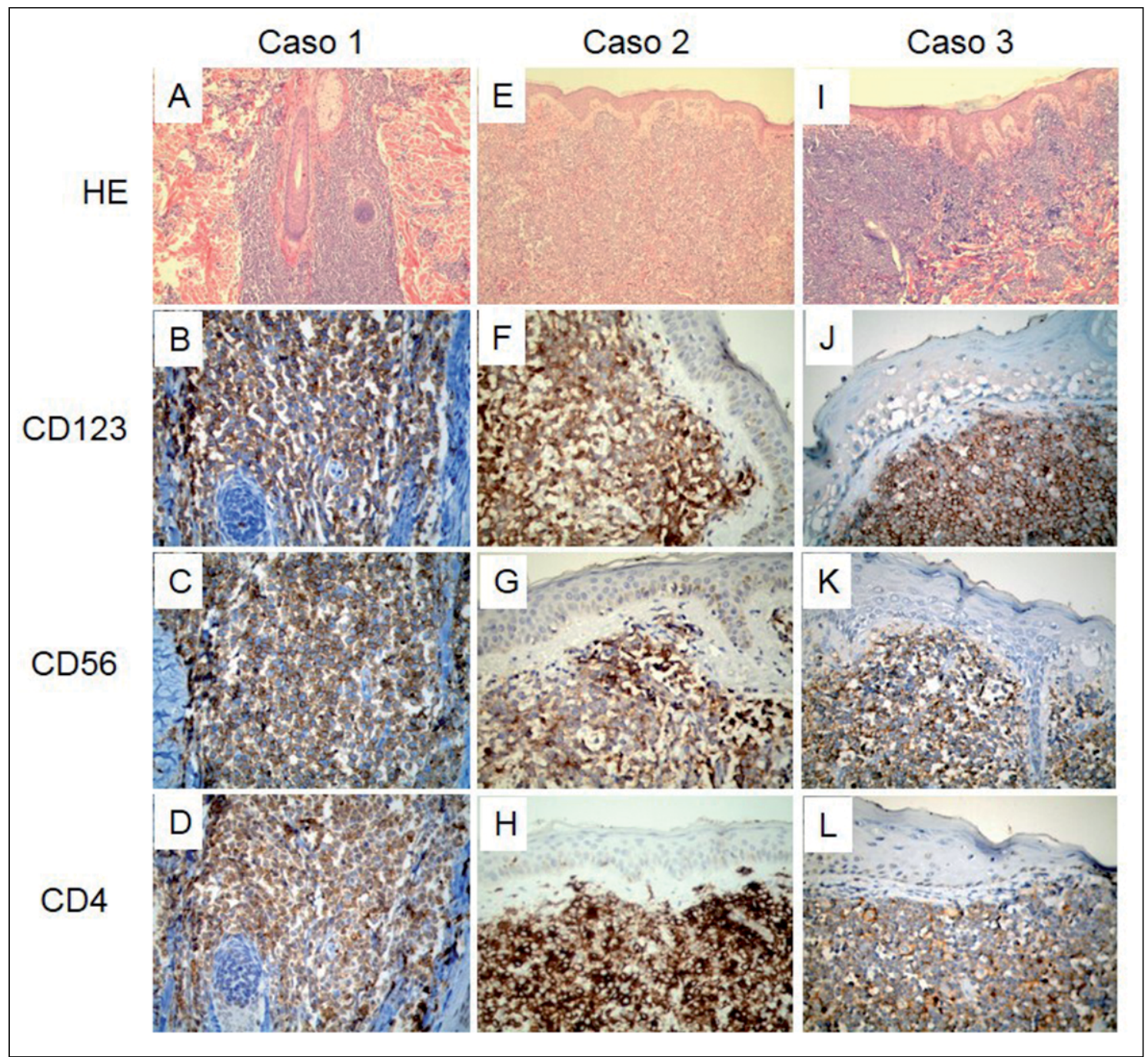

Figura 1. Biopsia de piel infiltrada por neoplasia maligna no epidermotrópica de aspecto blástico con patrón de crecimiento predominantemente perianexial. Las células neoplásicas tienen atipia moderada, núcleos ovoides, cromatina fina, nucléolo poco aparente y citoplasma escaso mal definido (A) (Hematoxilina-eosina, 40X). Biopsia de nódulo cutáneo que muestra infiltración difusa de la dermis por células neoplásicas de núcleos ovoides y hendidos, nucléolo poco aparente y citoplasma moderado mal definido (E) (Hematoxilina-eosina, 40X). Biopsia de lesión cutánea de la región frontal con infiltración difusa de la dermis y tejido subcutáneo por células neoplásicas de aspecto blástico (I) (Hematoxilina-eosina, 40X). En los tres casos las células neoplásicas expresan de forma difusa e intensa marcadores de células dendríticas plasmocitoides: CD123 (B, F, J), CD56 (C, G, K) y CD4 (D, H, L) (Técnica de inmunohistoquímica 400X).

\section{Discusión}

La NBCDP se presenta comúnmente en hombres, tiene una relación hombre-mujer de $3: 1$ y afecta principalmente adultos entre los 60 a 70 años de edad, aun cuando se han informado casos en pacientes menores de 40 años $^{6-9}$. La mayoría de los pacientes se presentan con placas o nódulos cutáneos, que eventualmente progresan con involucro de la médula ósea (90\%). La afección extracutánea es común, e incluye los ganglios linfáticos regionales (40\%-50\%), esplenomegalia $(44 \%)$ y hepatomegalia $(42 \%)^{10,11}$. De acuerdo a la clasificación de Ann Arbor el 66\% de los pacien- 
tes son diagnosticados en estadio IV y sólo $24 \%$ en estadio I ${ }^{9}$. Los casos informados en esta serie fueron hombres, dos de ellos menores de 40 años de edad, $y$ todos presentaron como primera manifestación lesiones cutáneas múltiples, localizadas predominantemente en la cara, el cuello y el tórax.

La incidencia exacta de la NBCDP se desconoce; representa aproximadamente $0,5 \%$ de las neoplasias hematológicas ${ }^{12}$. Hoy en día la etiología permanece desconocida; sin embargo, la exposición previa a quimioterapia y la asociación con neoplasias mieloides, sugiere la participación de posibles mutaciones en progenitores mieloides/ linfoides comunes o en células tallo hematopoyéticas $^{13,14}$.

Desde el punto de vista morfológico las lesiones cutáneas típicamente infiltran la dermis con distribución perivascular y/o perianexial, sin afectar la epidermis y conforme la enfermedad progresa se extiende hasta el tejido subcutáneo. Se caracteriza por una población monótona de células de pequeño a mediano tamaño con núcleos de contornos irregulares, cromatina fina, nucléolos pequeños y citoplasma en cantidad escasa a moderada.

En el estudio de IHQ, las células neoplásicas típicamente expresan CD4, CD56 y CD123; así como el antígeno sanguíneo de célula dendrítica 2 (BDCA/CD303) y la molécula dependiente de interferón $\alpha^{4,15}$.

Los estudios citogenéticos han informado perdidas genéticas que involucran 5 q21 o 5 q34, 12p13, 13q13-q21, 6q23, 15q; así como la pérdida completa del cromosoma $9^{11}$ y la translocación $\mathrm{t}(6 ; 8)(\mathrm{p} 21: \mathrm{q} 24)^{16}$. En la presente serie en uno de los pacientes se documentó monosomía del cromosoma 13 (-13q14.3), siendo esta una de las perdidas genéticas informadas con mayor frecuencia y que podría desempeñar un papel importante en la transformación maligna ${ }^{14}$.

El diagnóstico de NBCDP se basa en las características clínicas, los hallazgos morfológicos, el perfil de IHQ y los estudios citogenéticos; sin embargo, es importante considerar como diagnóstico diferencial: sarcoma mieloide, linfoma/ leucemia linfoblástico de células $\mathrm{T}$, linfoma de células T/NK tipo nasal extranodal y linfoma periférico de células $\mathrm{T}$.

Debido a la escasa información prospectiva, no hay un tratamiento estándar para estos pacientes. El tratamiento con dosis altas de HiperCVAD ha mostrado porcentajes de respuesta superiores a $90 \%{ }^{17}$. Por otro lado, esquemas de tratamiento tipo CHOP (ciclofosfamida, doxorrubicina, vincristina, prednisona), así como esquemas basados en ifosfamida/etopósido han logrado porcentajes de respuesta completa de $40 \%$ al $50 \%{ }^{18}$.

A pesar de que la NBCDP puede aparecer inicialmente como un tumor cutáneo localizado, podría considerarse de manera inicial un tratamiento agresivo incluyendo el TAloCPH. Actualmente se considera la mejor opción el TAloCPH durante la primera remisión completa, aumentando la supervivencia global y supervivencia libre de enfermedad, sin recaídas dentro de los primeros 27 meses $^{18,19}$. En nuestros pacientes el tratamiento consistió en HiperCVAD seguido de TAloCPH con una media de seguimiento de 14,6 meses. Al momento del estudio el primer paciente había fallecido con enfermedad, el segundo se encontraba en recaída documentada en piel y médula ósea y el tercero estaba vivo sin evidencia de enfermedad neoplásica. El paciente que falleció tenía factores de mal pronóstico: 51 años de edad, afección extracutánea al momento del diagnóstico, monosomía del cromosoma 13 (-13q14.3) y recaída en el día +178 posTAloCPH. Desafortunadamente, el pronóstico de los pacientes con NBCDP continua siendo pobre, con una supervivencia media de 12 a 19,8 meses, independientemente del tipo de tratamiento utilizado ${ }^{3,20}$.

En conclusión, es importante considerar la NBCDP como parte del diagnóstico diferencial de neoplasias hematológicas cutáneas. Son necesarios más estudios que nos permitan definir las características moleculares y marcadores biológicos de esta entidad, además de ensayos clínicos y estudios multicéntricos para establecer las estrategias terapéuticas óptimas que pudieran mejorar el pronóstico de estos pacientes.

\section{Referencias}

1. Petrella T, Bagot M, Willemze R, Beylot-Barry M, Vergier B, Delaunay $\mathrm{M}$, et al. Blastic NK-cell lymphomas (agranular CD4+ CD56+ hematodermic neoplasms): A review. Am J Clin Pathol 2005; 123 (5): 662-75.

2. Suzuki R, Nakamura S, Suzumiya J, Ichimura K, Ichikawa $\mathrm{M}$, Ogata $\mathrm{K}$, et al. Blastic natural killer cell lymphoma/leukemia (CD56-positive blastic tumor). Prognostication and categorization according to anatomic sites of involvement. Cancer 2005; 104 (5): 1022-31. 
3. Piña-Oviedo S, Ortiz-Hidalgo C. Neoplasia hematodérmica CD4+/CD56+. Diagnóstico histopatológico, fisiopatología y avances recientes de un tumor originado en células dendríticas plasmocitoides. Rev Esp Patol 2007; 40 (1): 11-22.

4. Facchetti F, Cigognetti M, Fisogni S, Rossi G, Lonardi S, Vermi W. Neoplasms derived from plasmacytoid dendritic cells. Mod Pathol 2016; 29 (2): 98-111.

5. Herling M, Jones D. CD4+/CD56+ hematodermic tumor: The features of an evolving entity and its relationship to dendritic cells. Am J Clin Pathol 2007; 127 (5): 687-700.

6. Dietrich S, Andrulis M, Hegenbart U, Schmitt T, Bellos F, Martens UW, et al. Blastic plasmacytoid dendritic cell neoplasia (BPDC) in elderly patients: results of a treatment algorithm employing allogeneic stem cell transplantation with moderately reduced conditioning intensity. Biol Blood Marrow Transplant 2011; 17 (8): 1250-4.

7. Feuillard J, Jacob MC, Valensi F, Maynadié M, Gressin $\mathrm{R}$, Chaperot L, et al. Clinical and biologic features of CD4+CD56+ malignancies. Blood 2002; 99 (5): 155663.

8. Bekkenk MW, Jansen PM, Meijer CJ, Willemze R. $\mathrm{CD} 56+$ hematological neoplasms presenting in the skin: a retrospective analysis of 23 new cases and 130 cases from the literature. Ann Oncol 2004; 15 (7): 1097-108.

9. Reimer $\mathrm{P}$, Rüdiger $\mathrm{T}$, Kraemer $\mathrm{D}$, Kunzmann $\mathrm{V}$, Weissinger F, Zettl A, et al. What is CD4+ CD56+ malignancy and how should it be treated? Bone Marrow Transplant 2003; 32 (7): 637-46.

10. Chan JK, Sin VC, Wong KF, Ng CS, Tsang WY, Chan $\mathrm{CH}$, et al. Nonnasal lymphoma expressing the natural killer cell marker CD56: a clinicopathologic study of 49 cases of an uncommon aggressive neoplasm. Blood 1997; 89 (12): 4501-13.

11. Liang X, Graham DK. Natural killer cell neoplasms. Cancer 2008; 112 (7): 1425-36.

12. Bueno C, Almeida J, Lucio P, Marco J, García R, de Pablos JM, et al. Incidence and characteristics of CD4+/
HLA DRhi dendritic cell malignancies. Haematologica 2004; 89 (1): 58-69.

13. Pagano L, Valentini CG, Pulsoni A, Fisogni S, Carluccio $\mathrm{P}$, Mannelli F, et al. Blastic plasmacytoid dendritic cell neoplasm with leukemic presentation: an Italian multicenter study. Haematologica 2013; 98 (2): 239-46.

14. Khoury JD, Medeiros LJ, Manning JT, Sulak LE, Bueso-Ramos C, Jones D. CD56+ TdT+ blastic natural killer cell tumor of the skin: a primitive systemic malignancy related to myelomonocytic leukemia. Cancer 2002; 94 (9): 2401-8.

15. Assaf C, Gellrich S, Whittaker S, Robson A, Cerroni $\mathrm{L}$, Massone $\mathrm{C}$, et al. CD56-positive haematological neoplasms of the skin: a multicentre study of the $\mathrm{Cu}$ taneous Lymphoma Project Group of the European Organisation for Research and Treatment of Cancer. J Clin Pathol 2007; 60 (9): 981-9.

16. Nakamura $\mathrm{Y}$, Kayano H, Kakegawa E, Miyazaki H, Nagai T, Uchida Y, et al. Identification of SUPT3H as a novel $8 \mathrm{q} 24 / \mathrm{MYC}$ partner in blastic plasmacytoid dendritic cell neoplasm with $\mathrm{t}(6: 8)(\mathrm{p} 21 ; \mathrm{q} 24)$ translocation. Blood Cancer J. 2015; 5 (4): e 301. doi:10.1038/ bcj.2015.26.

17. An HJ, Yoon DH, Kim S, Shin SJ, Huh J, Lee KH, et al. Blastic plasmacytoid dendritic cell neoplasm: a single-center experience. Ann Hematol 2013; 92 (3): 351-6.

18. Prochaska L, Dakhil C, Mathur S. Blastic plasmacytoid dendritic cell neoplasm: A rapidly progressive and fatal disease without aggressive intervention. Clin Med Insights Case Rep 2013; 6: 201-4.

19. Male HJ, Davis MB, McGuirk JP, Abhyankar S, Aljitawi OS, Zhang D, et al. Blastic plasmacytoid dendritic cell neoplasm should be treated with acute leukemia type induction chemotherapy and allogeneic stem cell transplantation in first remission. Int J Hematol 2010; 92 (2): 398-400.

20. Cui XB, Jin J, Pang XL, Li S, Liu CX, Li TT, et al. A case of blastic plasmacytoid dendritic cell neoplasm with ecchymotic lesions on the whole body. Int J Clin Exp Pathol 2014; 7 (7): 4391-9. 